\title{
Apport et stratégies de recouvrement de l'impôt sur les véhicules dans le budget de l'Etat Congolais.
}

\author{
Par Eder Mbala Kazadi*
}

\section{Résumé :}

Cette étude porte, telle que l'indique son intitulé, sur l'impôt sur les véhicules institué, conformément à la Constitution en vigueur, par la l'Ordonnance-loi n $13 / 001$ du 23 février 2013 fixant la nomenclature des impôts, droits, taxes et redevances des provinces et des entités territoriales décentralisées ainsi que leurs modalités de répartition. Ensuite, il est encadré par l'Edit n0002/08 du 22 janvier 2008 portant procédures relatives aux impôts, taxes, redevances et autres droits dus à la ville de Kinshasa et l'Edit n ${ }^{\circ} 0005 / 08$ du 11 octobre 2008 relatif aux impôts, taxes et droits provinciaux dus à la Ville de Kinshasa. Dans ce sens, la réflexion développée est non seulement centrée sur l'analyse de ce cadre juridique mais elle aborde aussi quelques aspects factuels relevés dans la mise en application dudit impôt que l'on qualifie abusivement de «vignette » dans le langage courant. A la fin de quoi, elle propose la revue de stratégies que requiert actuellement le recouvrement de cet impôt auprès de ses assujettis en vue de rendre son imposition plus significative et, par delà, permettre le renforcement du système fiscal congolais qui, concrètement, devrait être plus incitatif, persuasif qu'uniquement auto déclaratif et liquidatif pour le renflouement de la caisse budgétaire de la Ville Province de Kinshasa et par ricochet celle de l'Etat.

\begin{abstract}
:
This study relates, as its title indicates, to the vehicle tax instituted, in accordance with the Constitution in force, by Ordinance-Law No. 13/001 of 23 February 2013 fixing the tax nomenclature, duties, taxes and fees of the provinces and decentralized territorial entities as well as their distribution methods. Then, it is framed by the Edict $n{ }^{\circ} 0002 / 08$ of January 22,2008 relating to procedures relating to the taxes, duties, fees and other rights due to the city of Kinshasa and the Edict $n{ }^{\circ} 0005 / 08$ of October 11, 2008 relating provincial taxes, duties and fees due to the City of Kinshasa. In this sense, the reflection developed is not only focused on the analysis of this legal framework but it also addresses some factual
\end{abstract}

* L'auteur est Assistant à l'enseignement universitaire (Université de Kikwit et Université de Kinshasa/RDC). Il est également apprenant en 3è Cycle/DES-DEA en Droit/UNIKIN et Avocat inscrit au Barreau près la Cour d'appel de Kinshasa/Gombe. Contacts : edermbalakazadi@gmail.com/(+243)812467809.The author is a University Teaching Assistant (University of Kikwit and University of Kinshasa / DRC).He is also a 3rd Cycle student/DES-DEA in Law/UNIKIN and a lawyer registered with the Bar at the Kinshasa/Gombe Court of Appeal.Contacts: edermbalakazadi@gmail.com/(+243)812467809. 
aspects identified in the implementation of the said tax which is improperly described as a " sticker » in everyday language. At the end of which, it proposes the review of strategies that currently requires the collection of this tax from its taxpayers in order to make its taxation more significant and, beyond that, allow the strengthening of the Congolese tax system which, in concrete terms, should be more incentive, persuasive than only self-declaration and liquidation for the bailout of the budgetary fund of the City Province of Kinshasa and by extension that of the State.

\section{INTRODUCTION}

A l'instar de tous les Etats du monde, la République démocratique du Congo fait quotidiennement face à une gamme variée des charges d'intérêt général. Certaines d'entre elles sont requises pour la sauvegarde de la souveraineté nationale et d'autres se rapportent aux besoins du développement national ${ }^{1}$. Dans ce sens, la RDC fait recours à l'impôt en tant que ressource première et ordinaire ou même classique de tout Etat.

BALTHUS note, à cet effet, que : «tout citoyen d'un Etat doit contribuer au soutien du gouvernement dans la proportion la plus juste exactement mesurée par le revenu dont chacun d'eux jouit sous la protection du gouvernement $»^{23}$. C'est dans ce contexte que même ceux qui utilisent les véhicules doivent s'inscrire dans cette logique afin de renflouer la caisse de l'Etat.

Et comme cela est le moyen de financement de l'Etat, Maurice DUVERGER estime que c'est ça même les finances publiques, qu'il définit comme étant une science des moyens par lesquels l'Etat et les autres collectivités publiques se procurent et utilisent les ressources nécessaires à la couverture des dépenses publiques, par la répartition entre individus des charges qui en résultent ${ }^{3}$.

Cependant, c'est depuis 2013 que ce type d'impôt sur les véhicules est imposé aux utilisateurs des véhicules appelés à contribuer aux charges de la ville de Kinshasa par le truchement de la vignette. Et il s'y constate, d'une part, une mauvaise application de la loi fiscale par la Direction Générale de Recettes de Kinshasa (DGRK) et, d'autre part, on fustige également à Kinshasa la lourdeur et la multiplicité des perceptions d'impôt au sujet du même objet concerné qui est : le véhicule. De plus, on se plaint du manque des bons rapports qu'il y a avec les agents du fisc (DGRK), chaque fois que ceux-ci sont en contact avec leurs assujettis.

Pendant que la Ville Province de Kinshasa ne cesse d'enregistrer d'année en année des déficits budgétaires chroniques entrainant des conséquences dues notamment à l'absence

1 Voir BUABUA wa KAYEMBE Mathias, « Le droit fiscal congolais et son avenir », in Revue de la Faculté de droit, $2^{\text {ème }}$ année, Numéro 2, 2001, p. 265.

2 Voir UMBA Di MAMONA Achille, «Piste pour une fiscalité égalitaire en République démocratique du Congo », in Revue africaine de la démocratie et de la gouvernance (RADG), Institut pour la démocratie, la paix et le développement en Afrique (IDGPA), volume I, n4, 2014, p. 85.

3 Voir DUVERGER Maurice, «Finances publiques », Paris, 1988, Collec. Thémis, p. 15. 
des mécanismes de recevabilité et de communication de la part de l'administration fiscale sur les réalisations ou les services rendus grâce aux recettes réalisées, la méfiance du contribuable vis-à-vis du fisc, etc. De ce fait, cette entité provinciale semble ne pas tirer suffisamment profit des recettes issues de l'utilisation des véhicules sur son étendue au regard des nouvelles stratégies de recouvrement de l'impôt y relatif.

Ainsi, on peut s'interroger au sens de savoir : comment peut-on renforcer les mécanismes de recouvrement de l'impôt sur les véhicules au regard de son cadre juridique et des tracasseries rencontrées couramment en vue d'en rentabiliser l'apport dans le budget de l'Etat? Quels peuvent en être les stratégies et perspectives prévisionnelles susceptibles de mieux ressourcer l'Etat dans la mobilisation de recettes et dépenses publiques?

Ce dont la présente réflexion va tenter d'aborder, dans une démarche empirique, l'examen, par l'exégèse, du sujet titré en haut en quatre points suivants : d'abord le cadre juridique de l'impôt sur les véhicules (A), les contours essentiels de l'impôt sur les véhicules (B), ensuite, les stratégies actuelles de recouvrement (C), enfin, les perspectives pour un recouvrement réussi (D).

\section{A. LES ASPECTS JURIDIQUES DE RECOUVREMENT DE L'IMPOT SUR LES VEHICULES.}

La Constitution en vigueur en RDC dispose que : « sans préjudice des autres dispositions de la présente constitution, les matières suivantes sont de la compétence exclusive des provinces : (...) les impôts, taxes et droits et locaux notamment l'impôt foncier, l'impôt sur les revenus locatifs et l'impôt sur les véhicules automoteurs ${ }^{4}(\ldots) »$. Ceci revient à dire que l'impôt sur les véhicules est un impôt provincial par opposition aux impôts à caractère national et local.

Ainsi, Gaston JEZE voit dans l'impôt une prestation de valeur pécuniaire, exigée des individus d'après des règles fixes, en vue de couvrir des dépenses d'intérêt général et uniquement en raison du fait que les individus qui doivent le payer sont membres d'une communauté politique organisée ${ }^{5}$.

C'est dans ce sillage que l'article $1^{\text {er }}$ de l'Ordonnance-loi $n^{\circ} 13 / 001$ du 23 février 2013 qui a modifié et complété celle n69-006 du 10 février 1969 en la même matière dispose que : " la présente ordonnance-loi fixe la nomenclature des impôts, droits, taxes et redevances des provinces et des entités territoriales décentralisées et leurs modalités de répartition.

4 Voir l'article 204, al.16 de la Constitution de la République démocratique du Congo du 18 février 2006, modifiée par la loi ns ${ }^{\circ} 11 / 002$ du 20 janvier 2011 portant révision de certains articles de la constitution de la République démocratique du Congo.

5 Voir JEZE Gaston, "Cours de finances publiques », Paris, 1923-1930, p.3 54 : selon lui, il faut avoir six éléments essentiels, irréductibles de l'impôt moderne, à savoir : une prestation pécuniaire, sans contrepartie, un paiement forcé, des règles fixes, une destination d'utilité publique et enfin, l'impôt dû par des individus. 
Les règles de perception des impôts, droits, taxes et redevances provinciaux et locaux visés à l'alinéa $1^{\text {er }}$ sont fixés par voie d'édits ou des décisions des organes délibérants, conformément à la législation nationale ${ }^{6} »$. Pour ainsi dire que l'impôt sur les véhicules, appelé dans le langage courant : « vignette », fait l'objet de plusieurs édits. C'est-à-dire, chaque province devrait en prendre un.

C'est ainsi qu'il avait été pris l'édit de 2008 qui, en son article 14, dispose que : " l'impôt sur les véhicules est assis sur les véhicules admis à circuler sur l'étendue de la ville de $\mathrm{Kinshasa}^{7}$ ». C'est donc cet édit qui constitue le sous-bassement de la vignette pour la ville de Kinshasa.

Mais sans que les procédures de recouvrement dudit impôt soient prévues dans l'économie générale du texte. Ce qui donc a justifié la prise de l'édit du 22 janvier 2008 portant procédures relatives aux impôts, taxes, redevances et autres droits dus à la Ville de Kinshasa qui dispose que : «l'impôt sur les véhicules est dû par les personnes physiques ou morales qui utilisent un ou plusieurs véhicules ${ }^{8}$ ", ce qui est tout à fait logique comme l'atteste Michel BOUVIER qui conseille à tout Etat qu'afin de réaliser l'équité fiscal, le législateur doit toujours prendre en compte la capacité contributive du contribuable pour réaliser la justice fiscale ${ }^{9}$. Et donc, celui qui a une véhicule ne doit pas payer comme l'aurait fait celui qui en a deux ou plusieurs.

Voilà pourquoi, le redevable de l'impôt sur les véhicules souscrit une déclaration par véhicule, avant toute mise en circulation. Et il lui est délivré, pour ce faire une vignette qui atteste le paiement annuel sur les véhicules, et de la taxe spéciale de circulation routière ${ }^{10}$.

La déclaration doit être conforme au modèle arrêté par la Direction Générale de Recette de Kinshasa (DGRK) et est délivrée gratuitement au déclarant. C'est alors que la DGRK pourra procéder périodiquement au renouvellement partiel ou général des déclarations enregistrées et en distribuer, dans ce cas, les formulaires en temps opportun aux contribuables ${ }^{11}$.

Tel est l'essentiel du cadre légal de l'impôt sur les véhicules (vignette). Mais il en ressort quelques contours qu'il importe également d'évoquer ne fut-ce que l'essentiel.

6 Voir l'article $1{ }^{\text {er }}$ de l'Ordonnance-loi n ${ }^{\circ} 13 / 001$ du 23 février 2013 fixant la nomenclature des impôts, droits, taxes et redevances des provinces et des entités territoriales décentralisées ainsi que leurs modalités de répartition.

7 Voir l'Edit nº005/08 du 11 octobre 2008 relatif aux impôts, taxes et droits provinciaux dus à la Ville de Kinshasa.

8 Voir l'article 11 de l'édit $n^{\circ} 0002 / 08$ du 22 janvier 2008 portant procédures relatives aux impôts, taxes, redevances et autres droits dus à la ville de Kinshasa.

9 BOUVIER Michel, «Introduction au droit fiscal général et à la théorie de l'impôt », Paris, $11^{\text {ème }}$ édition, p. 13.

10 Voir l'article 12 de l'édit du 22 janvier 2008, note 8.

11 Voir l'article 13, note 8. 


\section{B. CONTOURS ESSENTIELS DE L'IMPOT SUR LES VEHICULES.}

Il convient de présenter avant tout ce qu'est-ce la notion (I) de l'impôt sur les véhicules après suivra sa matière imposable (II) ensuite, la base d'imposition (III).

\section{Notion :}

Selon les considérations d'ordre scientifique, l'impôt sur les véhicules ou la vignette est un impôt réel et direct par son essence. Et en vertu de l'Ordonnance-loi n69/006 du 10 février 1969 portant impôt réel telle que modifiée et complétée à ce jour, l'impôt réel est celui qui s'applique à un bien du fait qu'il existe et est source de revenu sans considération de la personne qui le supporte. Ceci atteste donc le caractère réel ${ }^{12}$ de la vignette.

Il est donc direct car, à travers le critère de la matière imposable, Roger KOLA GONZE pense que : " l'impôt direct est celui qui atteint périodiquement ou annuellement une matière imposable permanente qui se renouvelle régulièrement $»^{13}$.

Tirant conséquence de ces considérations tant légale que doctrinale, il peut être affirmé que l'impôt sur les véhicules ou la vignette est un impôt réel et direct, du fait qu'il atteint annuellement un bien (véhicules) et est source des revenus pour son utilisateur ou son propriétaire.

\section{Matière imposable :}

L'article 14 de l'édit de 2008 précité dispose que : « l'impôt sur les véhicules est assis sur les véhicules admis à circuler sur l'étendue de la Ville de Kinshasa ». ${ }^{14} \mathrm{Il}$ est clair que là il s'y trouve la matière imposable de la Vignette. Cet impôt frappe tous les véhicules à moteur sur l'étendue de la Ville de Kinshasa sous réserve de certaines exceptions.

Cependant, il est à remarquer que l'édit sus évoqué emploie le terme "véhicule » au lieu plutôt du terme « voiture ». Parce que, devra-t-on comprendre, le terme véhicule est à entendre ici en son plus large sens possible. Car, il ne désigne pas, selon Roger KOLA ${ }^{15}$, uniquement les véhicules automobiles, mais aussi tout engin de transport par terre ou par eaux quel que soit le moyen de propulsion utilisé en RDC.

Ce qui exclut automatiquement de ce cadre légal les avions et les aéronefs dont le cadre fiscal relève d'une autre législation n'intéressant pas la présente étude.

12 Voir les articles $1^{\text {eralinéa }} 2^{\text {ème }}$ et 40 alinéas 3èmes de l'ordonnance-loi $\mathrm{N}^{\circ} 69-006$ du 10 février 1969 sur l'impôt réel.

13 Voir KOLA GONZE Roger, "Cours de droit fiscal congolais », Faculté de droit, UNIKIN, 2018 2019, p. 26.

14 Voir l'article 14 de l'édit du 22 janvier 2008, note 8.

15 Voir KOLA GONZE Roger, note 14, p.27. 
Néanmoins, en l'espèce, c'est la notion de l'utilisation ${ }^{16}$ des véhicules qui est d'application. C'est-à-dire les personnes physiques ou juridiques qui utilisent un ou plusieurs véhicules doivent payer cet impôt. Le terme « utilisateur » trouve sons sens dans le fait que l'on soit propriétaire ou non, lorsqu'on use d'un véhicule, on est tenu dès lors de payer cet impôt.

Par ailleurs, l'article 14 sus évoqué connaît quelques limites à l'effet desquelles nous précisons que certains véhicules ne sont pas assujettis à cet impôt suite à leur appartenance à une quelconque catégorie des personnes. Raison pour laquelle, l'article 15 dudit édit dispose que : "les dispositions relatives aux exonérations, aux redevables, à la période imposable et à la débition de l'impôt sont celles prévues par l'Ordonnance-loi nº69/006 du 10 février 1969 relative à l'impôt réel telle que modifiée et complétée à ce jour $»^{17}$. Et en vertu de celle-ci, précisément son article $39^{18}$, il est ordonné que l'impôt sur les véhicules n'est pas établi en ce qui concerne :

1.Les véhicules appartenant à l'Etat, aux provinces, aux villes, aux communes, aux circonscriptions administratives, ainsi qu'aux offices et établissements publics de droit congolais n'ayant d'autres ressources que celles provenant de subventions budgétaires;

Les véhicules appartenant aux institutions, associations et établissements visés à l'article 2 de la présente ordonnance-loi;

1 Les véhicules appartenant aux Etats étrangers, et affectés exclusivement à l'usage d'agents ayant le statut d'agents diplomatiques. Cette exemption n'est consentie que sous réserve de réciprocité;

2 Les véhicules appartenant aux organismes internationaux et utilisés exclusivement pour les besoins desdits organismes;

3 Les véhicules appartenant aux membres du corps diplomatiques étrangers de même qu'aux consuls et agents consulaires accrédités au Congo, à la triple condition :

a) Qu'ils soient sujets d'Etat qu'ils représentent;

b) Que les gouvernements dont ils sont les mandataires accordent la même immunité aux agents diplomatiques et consulaires congolais;

c) Qu'en ce qui concerne les agents consulaires, ils n'exercent aucune activité professionnelle autre;

4 Les dépanneuses;

5 Les véhicules servant à la manutention, au transport ou à la traction dans l'enceinte des gares, des ports et des aérodromes;

6 Les cyclomoteurs d'une cylindrée n'excédant pas 50 centimètre cube;

7 Les véhicules à traction ou à propulsion humaine ou animale;

8 Les véhicules à moteur équipés spécialement pour l'extinction des incendies;

16 Voir l'article 11 de l'édit du 22 janvier 2008, note 8.

17 Voir l'article 15 de l'édit du 11 octobre 2008 relatif aux impôts, taxes et droits provinciaux et locaux dus à la ville de Kinshasa.

18 Voir l'article 39 de l'Ordonnance-loi n 69-006 du 10 février 1969 sur l'impôt réel. 
9 Les auto-ambulances et les véhicules utilisés exclusivement comme moyens de locomotion personnelle par les grands invalides ou par les infirmes;

10 Les machines-outils;

11 Les véhicules à moteur utilisés exclusivement à l'essai par les fabricants ou marchands ou par leurs employés;

12 Les véhicules utilisés par des personnes qui n'ont ni domicile, ni résidence, ni établissement au Congo, et qui n'y exercent aucune activité lucrative;

13 Les navires de mer voyageant au long cours et les navires employés au grand cabotage, c'est-à-dire tous ceux qui, dans leurs voyages périodiques, dépassent la latitude du cap Lopez ou celle du cap Frio;

14 Les voiliers. Ces engins ne sont pas légalement assujettis à l'impôt sur les véhicules ou la vignette, ils sont donc exonérés.

Ainsiprésenté, vérifions à présent au point suivant la base juridique de l'imposition ou de l'assujettissement des véhicules assujettis.

\section{LA BASE D'IMPOSITION :}

La base d'imposition est représentée par la catégorie, le poids et la puissance du véhicule ${ }^{19}$. Pour GUY GEST et Gilbert TIXIER, la base d'imposition c'est le socle même de l'imposition, et assoir l'impôt c'est définir ou plutôt délimiter la matière imposable, puis l'évaluer ${ }^{20}$.

C'est donc sur ces critères qu'est calculé le taux d'imposition même en RDC comme nous le verrons un peu plus loin. De ce fait, on peut déduire que l'impôt sur les véhicules est assis sur les engins admis à circuler sur l'étendue de la Ville de Kinshasa selon le contenu de l'édit de 2008 sus évoqué. Et de ce même édit ressort directement le fait générateur de cet impôt qui est la mise en circulation de l'engin automoteur.

Le cadre juridique étant ainsi examiné, voyons à présent ce qu'il en est des mécanismes de recouvrement.

\section{LES STRATEGIES DE RECOUVREMENT DE L'IMPOT SUR LES VEHICULES.}

Il convient de relever déjà que le recouvrement est la phase ultime de tout travail d'assiette. L'objet poursuivi est, en effet, d'obtenir que les fonds publics provenant de l'impôt entrent au Trésor Public au lieu indiqué, au moment convenu et en toute sécurité. ${ }^{21}$

19 Voir BELTRAME Pierre, «La fiscalité en France », Paris, 1993, p. 121.

20 Voir GUY GEST et GILBERT TIXIER, « Manuel de droit fiscal », Paris, 1986, p. 158.

21 Voir BUABUA wa KAYEMBE Mathias, « Traité de droit fiscal congolais », Kinshasa, 1993, p. 256. 
De ce fait, d'aucun n'hésite de s'interroger sur comment est recouvré cet impôt sur la Ville de Kinshasa. Roger KOLA GONZE rapporte que : « l'impôt sur les véhicules donne lieu à l'apposition d'un timbre fiscal sur les véhicules appelé vignette $»{ }^{22}$

Mais il est à noter qu'à cet effet dans le langage courant, l'impôt sur les véhicules est assimilé au timbre fiscal (vignette). Ce qui, à bien d'égards, peut prêter à confusion et être illogique, dans la mesure où, la vignette n'en est que la preuve de paiement de l'impôt sur les véhicules et non l'appellation de ce dont elle prouve le paiement. Puisque sémantiquement, le mot « vignette » signifie « étiquette » portant l'estampille de l'Etat qui certifie le paiement de certains droits, par exemple de l'impôt sur les automobiles ${ }^{23}$. Ce qui revient à dire que la vignette n'est pas l'impôt sur les véhicules.

En d'autres termes, si une somme d'argent quelconque est perçue pour la délivrance de la vignette, ladite somme ne devrait pas être considérée comme l'impôt mais plutôt comme une taxe administrative. Au sens où Mathias BUABUA wa KAYEMBE voit, en cette taxe administrative, un prix payé par un usager pour un service précis et déterminé qui lui est rendu par la collectivité publique. Et donne l'exemple de l'obtention d'un passeport, et conclut pour dire que la taxe administrative est aussi une rémunération pour service rendu, d'où le surnom de la taxe rémunératoire lui donné ${ }^{24}$.

Il s'en déduit donc qu'une taxe est liée à la prestation d'une contrepartie déterminée, ce qui n'est pas le cas de l'impôt dont la contrepartie n'est connue du contribuable.

Ainsi on peut supposer que si le frais est destiné à l'obtention d'une vignette, on parlerait d'une taxe. Par contre, le frais versé au trésor pour son véhicule devrait être perçu comme un impôt parce qu'admettre que l'impôt sur les véhicules est une taxe ferait relever d'une assertion illégale.

Ceci dit, on peut s'interroger maintenant sur comment se procure-t-on donc la vignette et quelles en sont les catégories concernées? Et quel mécanisme serait efficace pour contrer les assujettis récalcitrants qui s'adonnent à la fraude ou à l'évasion fiscale en utilisant en circulation des véhicules non déclarés?

\section{La procuration de la Vignette :}

Pour se procurer la Vignette, tout assujetti doit se présenter à une Agence d'un établissement bancaire de la place nommé «FBN Banque » pour les personnes physiques et/ou à un autre nommé «SOFI Banque » pour les personnes morales muni du document du véhicule auprès de l'agent de la DGRK qui, au vu des informations contenues dans le document, établit la note de calcul.

22 Voir KOLA GONZE Roger, note 14, p. 28.

23 Voir Dictionnaire le Larousse, édition 2015, p. 447.

24 Voir BUABUA wa KAYEMBA Mathias, note 22, p. 34. 
Une fois établie, la note de calcul est transmise au préposé de é-recette ${ }^{25}$ pour édit de la note de perception. Celle-ci est signée au nom de l'agent de la DGRK et remise à l'assujetti pour effectuer le paiement au guichet de la Banque.

Après l'obtention de cette preuve de paiement, l'assujetti peut alors se présenter auprès du préposé de é-recette pour que celui-ci lui délivre un Certificat de Paiement Informatisé (CPI), titre obligatoire pour l'impression de la vignette informatisée. Celle-ci est ainsi mise à disposition du comptable principal des matières ou son subordonné pour délivrance à l'assujetti.

Mais en cas de perte de la vignette, il est possible que l'assujetti se présente avec son Certificat de Paiement Informatisé au préposé de la DGRK qui pourra lui faciliter l'obtention d'une nouvelle vignette moyennant paiement de $30 \%$ du coût global de la vignette.

Par contre, en cas de perte de la vignette et du Certificat Informatisé de Paiement, le requérant est sommé de payer la totalité de ce que coûte la vignette pour qu'une nouvelle lui soit délivrée. Telle est la procédure à suivre fournie par le Directeur Général de la DGRK $^{26}$.

Par ailleurs, tous les véhicules n'étant pas d'un même poids, catégorie et puissance du moteur comme BELTRAME le rapporte ci-dessus dans le calcul de paiement, le Directeur Général de la DGRK a dû lui classifier comme suit les véhicules en rapport avec le paiement de la vignette.

\section{Classification des véhicules et mode de paiement27 :}

Les assujettis personnes physiques, propriétaires des véhicules utilitaires de moins de 2.500 kgs sont sommés de payer 72,4 dollars ou l'équivalent en franc congolais. Pour les mêmes engins, les personnes morales paient 89,1 dollars. Les véhicules utilitaires pesant entre 2.500 et $10.000 \mathrm{Kgs}$ devront payer 75,6 dollars pour les personnes physiques et 97,6 dollars pour les personnes morales.

Pour les véhicules utilitaires de plus de $10.000 \mathrm{Kgs}$, les propriétaires personnes physiques sont obligés de payer 83,5 dollars, les personnes morales paient 97,4 dollars.

Les propriétaires de véhicules de tourisme de 1 à 10 chevaux vapeurs (cv) devront débourser 79,5 dollars pour les personnes physiques et 81,5 dollars pour les personnes morales.

Pour les véhicules de 11 à $15 \mathrm{cv}$, la vignette coûte 88 dollars pour les personnes physiques et 91,5 dollars pour les personnes morales. Les vignettes pour les véhicules de

25 Ce sont des agents recrutés par la Direction générale des recettes de Kinshasa et commis auprès des banques désignées aux fins d'imprimer et de délivrer les certificats de paiement informatisé, et donc, ils dépendent de la DGRK mais attachés à chaque banque pour des besoins d'harmonisation et d'informatisation des données de la Direction.

26 Voir Jean Paul BILANGA N'OSHUM, Directeur général de la direction générale des recettes de Kinshasa, in https://www.google.com/lepotentielonline. Consulté le 20 novembre 2020. ${ }^{27}$ Voir Ibidem. 
tourisme de plus de $15 \mathrm{cv}$ coûtent 97,8 dollars ou leur équivalent en franc congolais pour les personnes physiques et 101,6 dollars pour les personnes morales.

Les véhicules tracteurs de 1 à $10 \mathrm{cv}$ paient 63 dollars, 73,1 dollar pour les tracteurs de 10 à $15 \mathrm{cv}$ et les tracteurs de plus de $15 \mathrm{cv}$ paient 83,1 dollar, payables également en franc congolais.

Les véhicules remorques de moins de $2.500 \mathrm{Kgs}$ sont obligés de payer 83,1 dollar et ceux de $2.500 \mathrm{Kgs}$ à $10.000 \mathrm{Kgs}$ paient 93,2 dollars pour les personnes physiques comme pour les personnes morales.

A ce sujet, il est à noter que ces taux de taxation c'est pour l'obtention de la vignette. C'est pourquoi, il est convenable de dire « taux de taxation » et non " d'imposition ». Ceci ne doit pas être confondu avec le paiement de l'impôt sur les véhicules lequel obéit au principe de la légalité de l'impôt.

Car, le montant ou taux de l'impôt sur les véhicules est précisément fixé par la loi. C'est sous cette houlette que l'article 16 de l'édit du 11 octobre 2008 relatif aux impôts, taxes et droits provinciaux et locaux dus à la Ville de Kinshasa dispose que : le taux de l'impôt sur les véhicules est fixé comme suit ${ }^{27}$ :

1. Motocycles : l'équivalent en franc congolais de 10 dollars;

2. Véhicules automobiles utilitaires :

a) De moins de $2500 \mathrm{~kg}$ : équivalent en franc congolais de 15 dollars,

b) De moins de $2500 \mathrm{~kg}$ à $10.00^{\circ} \mathrm{kg}$ : équivalent en franc congolais de 20 dollars,

c) De moins de $10.000 \mathrm{~kg}$ : équivalent en franc congolais de 23 dollars;

3. Véhicules de tourisme :

a) Appartenant aux personnes physiques :

- De 01 à 10 chevaux-vapeurs : équivalent en franc congolais de 20 dollars,

- De 11 à 15 chevaux-vapeurs : équivalent en franc congolais de 23 dollars,

- De plus de 15 chevaux-vapeurs : équivalent en franc congolais de 27 dollars;

b) Appartenant aux personnes morales :

- De 01 à 10 chevaux-vapeurs : équivalent en franc congolais de 30 dollars,

- De 10 à 15 chevaux-vapeurs : équivalent en franc congolais de 35 dollars,

- De plus de 15 chevaux-vapeurs : équivalent en franc congolais de 50 dollars;

4. Véhicules tracteurs imposables, selon le cas, aux taux prévus sous les litteras B ou C ci-dessus;

a) Bateaux et embarcations à propulsion mécanique servant exclusivement ou accessoirement au transport de personnes : l'équivalent en franc congolais de 6 dollars par cheval vapeur;

27 Voir l'article 16 de l'édit $n^{\circ} 0005 / 08$ du 11 octobre 2008 relatif aux impôts, taxes et droits provinciaux et locaux dus à la ville de Kinshasa. 
b) Bateaux et embarcations à propulsion mécanique servant exclusivement au transport de marchandises, au remorquage ou au touage : l'équivalent en franc congolais de 4 dollar par cheval vapeur;

5. Baleinière, barges et autres embarcations remorquées : l'équivalent en franc congolais en franc congolais de 9 dollars par mètre cube de jauge nette indiquée au certificat de jaugeage;

6. Bateaux et embarcations de plaisance à propulsion mécanique : équivalent en franc congolais de 17 dollars par cheval vapeur.

Par ailleurs, il convient de signaler qu'à l'heure actuelle dans la ville de Kinshasa, l'achat de vignette ou le paiement de l'impôt sur les véhicules fait l'objet des nouvelles stratégies de recouvrement qui sont essentiellement l'ouverture des comptes dans les deux banques susmentionnées pour le compte du trésor (FBN Banque et SOFI Banque).

En dépit de tous les efforts fournis par les autorités compétentes pour une nouvelle politique fiscale en la matière, il est toujours déplorable de remarquer que la plupart des assujettis à cet impôt ne s'acquittent pas de leurs obligations citoyennes, moins encore ils n'achètent pas la vignette d'une façon régulière à Kinshasa. Ce qui occasionne également, faudra-t-il le dire, de façon récurrente et malencontreuse la pénurie de transports en commun à chaque recouvrement forcé sur les artères de la Ville de Kinshasa.

\section{LES RECETTES REALISEES AU BUDGET DE LA VILLE DE KINSHASA POUR L'EXERCICE 2019 ET 2020 :}

Deux dernières années nous ont servi d'échantillon pour évaluer l'apport concret de la vignette dans le ravitaillement du budget de la ville province de Kinshasa, obtenues grâce à l'entretien avec un cadre de la DGRK qui a requis l'anonymat étant donné que les informations obtenues risquaient d'entraver à son travail au regard de leur nature critique. En voici :

\section{a. Pour 2019, l’impôt sur les véhicules a été perçu de la manière suivante :}

\section{- Pour les personnes morales}

- Il y a eu 1.015 véhicules de transport en commun immatriculés, et l'impôt perçu s'élevait à 17.618 dollars américains;

- 8 Véhicules immatriculés, ayant eu comme valeur contributive 482, 4 dollars américains

- Véhicules exonérés et exemptés : 830, alors l'Etat a perdu 12.450 dollars américains

- Total : 364 qui donne la valeur de 411, 12 dollars américains; -Total pp= 5607

$-=284168,38$

\section{Unités flottantes}

a). 69 barques ont flotté sur les rives congolaises et ont contribué au budget de la ville province de Kinshasa à la hauteur de 7.592.310 dollars américains; 
b). 11 bateaux ont flotté également les rives congolaises et ont ravitaillé la ville province à la hauteur de 1. 703, 4 dollars américains.

\section{- Pour les personnes physiques}

Il y a eu :

- 28 motos légalement immatriculées, et ont fourni 392 dollars au budget de la ville province;

- 200 véhicules auto, et l'administration fiscale de la ville province (DGRK) a récolté 11. 250 dollars américains;

- 4. 951 véhicules de tourisme, ayant contribué à la hauteur de 235. 122, 18 dollars américains;

- 428 tracteurs et remorques, ayant contribué à la hauteur de 17. 403, 9 dollars américains;

- 48 bateaux à propulsion ayant fourni 5.214 dollars américains

Total : $228=14.509,7$ dollars américains

Total général : $14.33=663.089,2$ dollars américains

\section{b. Pour l'an 2020, l'impôt sur les véhicules a été perçu de la manière suivante :}

Il y a eu :

- 283 motos, et ont contribué à la hauteur de 3.611 dollars américains;

- 2.064 véhicules utilitaires, ayant fourni 144.560 dollars américains;

- 5450 véhicules touristiques, ayant ravitaillé le budget de la vile province de Kinshasa à la hauteur de 286.885 dollars américains :

- 911 tracteurs et remorques, ont contribué à la hauteur de 43. 255, 34 dollars américains. ${ }^{28}$

Ces chiffres sombres, paraissent inquiétant au regard de l'engouement des engins sur les rues de Kinshasa, les routes se dégradent du jour au lendemain, tout le monde crie à la mauvaise qualité construction, mais personne ne sait s'interroger sur cette fraude fiscale, car à l'issue de ces récoltes que le FONER (Fond d'Entretien Routier) parvient à entretenir lesdites routes. Mais rien ne va, personne ne veut immatriculer son véhicule, et les agents commis à cet effet ne fournissent aucun effort dans les réformes de l'administration ou recouvrement de cet impôt.

D'où, pour notre part, il s'est avéré judicieux de pouvoir réfléchir de nouveaux sur les stratégies du recouvrement de cet impôt, car les préjudices du défaut de son recouvrement ou son recouvrement irrégulier, n'épargnent personne. C'est donc cette pensée qui nous conduit au point dont la teneur suit :

D'où, l'occasion de penser à des nouvelles perspectives.

28 Données obtenues auprès d'un Agent de la Direction Générale des Recettes de Kinshasa (DGRK) qui a requis l'anonymat en date du lundi 05 avril 2021, au siège de la Direction. 


\section{E. LES PERSPECTIVES POUR LA RENTABILITE DU RECOUVREMENT DE L'IMPOT SUR LES VEHICULES.}

L'impôt n'est accepté par les contribuables que s'il est adapté à leurs facultés contributives et que leurs charges ne sont pas excessives par rapport à celles des autres ${ }^{29}$. Dans la même logique IKAS note que : tout impôt doit être levé dans le temps et de la manière qui convient le mieux aux imposés. Il s'impose, en effet, de réduire au minimum le sacrifice, toute souffrance qui dépasse le strict nécessaire et l'illégal. ${ }^{30}$

C'est donc en cela que l'impôt sur le véhicule peut remplir sa fonction sociale dans la ville province de Kinshasa, comme l'a estimé Marc EROY : la fonction sociale de l'impôt se traduit par une redistribution des revenus qui peut être modulée en faveur ou en défaveur d'un groupe social. ${ }^{31}$

Dès lors, il convient de diagnostiquer les approches stratégiques susceptibles de pousser les assujettis à facilement souscrire et s'acquitter du devoir de paiement de cet impôt. Puisque les principales causes de ce malheureux constat sont connues et essentiellement dues, pensons-nous, aux mauvaises stratégies actuelles de recouvrement de l'impôt sur les véhicules que sont notamment la tracasserie et la lourdeur de notre système fiscal, le trafic d'influence, la culture fiscale déficitaire, la multiplicité de taux d'imposition sur un même véhicule, l'apathie et la corruption des services du fisc lors de contrôles fiscaux, etc. Mais il convient d'aborder en contre-proposition des solutions à envisager de lege ferenda.

\section{La réforme du système fiscal en vue d'éradiquer la tracasserie et la lourdeur systématiques :}

Par définition, la tracasserie est tout ce qui pose ennui, désagrément et problème ${ }^{32}$. UMBA Achille estime que : « le système fiscal mal connu des contribuables ne peut qu'engendrer l'incompréhension, et chaque contrôle ou visite des agents des impôts est qualifié tout de suite de racket et tracasserie.

Cette tracasserie prend des proportions importantes par le fait de la multiplication des contrôles et l'absence de communication ou d'explication claire des agents de l'administration fiscale qui incline aux contraintes diverses. Cette situation de tracasserie influe sur le comportement des citoyens. ${ }^{34}$

C'est donc cette situation qui pousse beaucoup d'utilisateurs des véhicules à ne pas payer l'impôt ni se procurer de la vignette à Kinshasa. Par exemple, en Allemagne, lorsque des sanctions sévères sont édictées par le législateur contre les fraudeurs, elles sont très

29 Voir UMBA Di MAMONA Achille, note 3, p. 84.

30 Voir UMBA Di MAMONA Achille, note 3, p. 86.

31 Voir EROY Marc, «La sociologie fiscale socio-legos, 2008 »,inRevue de l'association française de sociologie, disponible in http://socio-legos.revue.org.

32 Voir le Dictionnaire le Larousse, édition 2015, p. 447. ${ }^{34}$ Voir UMBA Di MAMONA Achille, note 3, p. 89. 
généralement appliquées; en Italie, il semble que bien souvent les magistrats soient portés à plus d'indulgence vis-à-vis des fraudeurs, et tendent à édulcorer les pénalités ${ }^{33}$.

Ainsi, plusieurs opérateurs économiques Congolais se lancent informellement, eux par contre, dans le secteur de transport pour se retrouver soit dans la fraude fiscale soit dans l'évasion fiscale. Ceci prouve que le système fiscal congolais en matière de l'impôt sur les véhicules et de la vignette est moins développé que ça soit tant par les assujettis que par les agents du fisc commis à cet effet. Ce qui conduit à la tracasserie qui ne facilite donc pas le paiement escompté.

Il ne se constate une récolte fiscale en matière de l'impôt sur les véhicules qu'au travers de l'achat de la vignette. Les contribuables s'obstinent généralement à ne pas payer l'impôt, et chaque fois que les agents du fisc arrivent pour contrôler, ils fuient ou renoncent aux différents rendez-vous, jusqu'à fatiguer les contrôleurs ${ }^{34}$.

Tolérer la récolte fiscale, atténuer les obligations de certains groupes sociaux sous leur menace directe, c'est porter atteinte à la notion du devoir fiscal dans l'esprit de l'assujetti. Car le fait de céder à des revendications présentées sous des formes inacceptables fait naître de nouvelles résistances et conduit à des nouveaux abandons. C'est pourquoi la réforme et l'effectivité de l'application de la loi fiscale en cette matière reste à suggérer en priorité.

\section{Le trafic d'influence :}

Il sied de noter que certains contribuables profitent de leurs statuts, de leurs positions économiques et financiers, de leurs fonctions ou de leurs relations informelles avec les autorités des instances supérieures pour intimider et contraindre les agents du fisc de ne pas exercer un suivi et un contrôle sur leurs véhicules.

Cette pratique doit être éradiquée par la conscientisation du citoyen à contribuer au fonctionnement de la ville de Kinshasa dans le domaine de transport, comme le prévoit CASTAGNE, l'impôt peut être utilisé pour favoriser un secteur d'activités particulier, et cela s'illustre par exemple dans le développement des entreprises ou bien encore l'aménagement du territoire ${ }^{35}$.

Donc, le paiement de la vignette peut favoriser à la ville province de maximiser les recettes et développer le secteur de transport qui semble ne pas être développé jusqu'à preuve du contraire.

33 Voir UMBA Di MAMONA Achille, note 3, p. 90.

34 Voir DERUEL, Finances publiques, droit fiscal, $6^{\text {ème }}$ édition, Paris, 1987, p.27.

35 Voir CASTAGNE Bernard, La politique fiscale, Que sais-je?, Paris, 2008, p. 5. 
Apport et stratégies de recouvrement de l'impôt sur les véhicules dans le budget de l'Etat Congolais.

\section{Le contrôle fiscal inversé et l'instauration du principe de transparence en faveur des assujettis :}

L'article 12 alinéa premier de l'édit du 22 février 2008 sus évoqué dispose que : le redevable de l'impôt sur les véhicules souscrit une déclaration par véhicule, avant toute mise en circulation $^{36}$. Cette disposition consacre le caractère déclaratif de notre système fiscal en cette matière.

A ce sujet BUABUA wa KAYEMBE pense que : " la déclaration est une simple confession, seule l'existence d'un contrôle fiscal rigoureux et systématique peut permettre sinon d'éradiquer, du moins de limiter les effets de la fraude fiscale ${ }^{37} »$. Prenant l'encontre, Pierre BELTRAME note, lui, que : " l'essentiel des recettes provient d'impôt déclaratif. Il est donc nécessaire que le fisc puisse vérifier les confessions fiscales souscrites par les contribuables $^{40} »$.

Le Guide fiscal de l'artiste plasticien argue que : tout système déclaratif a pour contrepartie la possibilité d'un contrôle ultérieur par l'administration, de la sincérité des déclarations $^{38}$. Au regard de toutes ces considérations doctrinales, il peut être compris qu'en vue d'une récolte escomptée de l'impôt sur les véhicules et de la vignette, l'administration fiscale en cette matière doit renforcer le dispositif de son personnel, mais surtout conscientiser ceux qui sont affectés auprès des banque pour cette fin. Et il est convenable de permettre aux contribuables la possibilité de contrôler inversement aussi le fisc en guise du principe de transparence de la bonne gouvernance.

A titre d'exemple, un journaliste rapporte que : le prix de la vignette a été fixé par le gouvernement provincial de Kinshasa selon la puissance du moteur de chaque véhicule et communiqué officiellement aux usagers. Et il varie entre 80 USD à plus de 100 USD mais curieusement aux agences de la FBN Banque et SOFI Banque, les seules retenues, pour ces opérations, les agents de la DGRK exigeraient 5000 FC par dossier, en plus du prix de la vignette qui leur est particulièrement rétrocédé par la Ville. Se trouvant sur l'Agence FBN Banque de KASA VUBU (Khartoum), le reporteur de Scooprdc.net a assisté à ce qui conviendrait d'appeler une méfia à ciel ouvert, confirmé par d'autres journalistes qui sont passés par cette agence voire le même constat à l'agence se trouvant sur 24 novembre tout comme à celle de SOCIMAT. ${ }^{39}$

Et à raison ou à tort, les agents de la DGRK justifient cette perception qui surprend tout assujetti qui vient payer, par la motivation de leur travail, qui du reste est pris en charge par l'hôtel de ville qui aura à rétrocéder à la DGRK sa part réglementaire. Cette somme

36 Voir l'article 12 alinéas 1ers de l'édit du 22 février 2008, note 8.

37 Voir BUABUA wa KAYEMBE Mathias, « Le droit fiscal congolais et son avenir », in Revue de la faculté de droit, $2^{\text {ème }}$ année $\mathrm{n}^{\circ} 2,2001$, le droit congolais face à son avenir, Université protestante du Congo (UPC), p. 274. ${ }^{40}$ Voir BERTRAME Pierre, La fiscalité en France, Paris, 1993, p. 138.

38 Voir« Guide fiscal de l'artiste plasticien », la documentation française, Paris, 1987, p.83.

39 Lire le contenu de ce témoignage fait par le journaliste DANNY MANGBAU in https://Scooprdc. net. 
indûment perçue en FC si elle est convertie en devise américaine donnerait un montant de 3,03 dollars par dossier. Ce qui ferait avec l'achat de 50 mille propriétaires des véhicules qui auront payé la vignette, la DGRK encaisserait 150.000 dollars en plus de la rétrocession normale dont est censée bénéficier le gouvernement provincial.

Ce qui, de plus, est déplorable c'est que dans le paiement de la vignette, le virement bancaire n'est pas accepté, alors qu'en ce temps moderne cette opération d'une banque à une autre facilite les transactions. Pourtant la numérisation pourrait bien faire éviter de fils d'attente dans des conditions précaires dont on observe devant les banques en cas de surnombre. ${ }^{40}$

C'est là la preuve de l'inefficacité de contrôle fiscal en matière de recouvrement fiscal. C'est ce qui pousse notamment l'Administration físcale à la défaillance.

DERUEL n'a-t-il pas tort lorsqu'il pense que « le contrôle recouvre l'ensemble des moyens dont dispose l'administration pour vérifier et garantir l'application correcte de la loi fiscale; il est assorti des garanties, de moyens de poursuite et des sanctions ${ }^{41} »$.

D'où, il importe de renforcer les mécanismes de contrôle a priori des agents par leurs supérieurs (contrôle hiérarchique), accompagné de la dotation des garanties aux assujettis (prise en compte de dénonciation), et des sanctions effectives et exemplaires sur tout agent récalcitrant d'abord afin que le contrôle puisse être finalement exercé de manière objective et efficace sur les assujettis.

\section{$V$. La restauration de la confiance entre les assujettis et l'administration fiscale par le mécanisme de caméras ambulants :}

Pour être réellement utile au pays, les services du fisc devraient manifester un activisme à toute épreuve dans le recensement des contribuables, la diffusion de l'information et des formulaires fiscaux, l'impression des avertissements-extrait de rôle et l'organisation des opérations de recouvrement. ${ }^{42}$

Car comme l'a affirmé Luc SAIDJ, il est incontestable que l'impôt qui constitue la part la plus important des recettes de l'Etat, est nécessaire pour financer ses dépenses et celles des collectivités territoriales ${ }^{43}$. Et donc, la vignette en tant qu'impôt doit servir à cette fonction budgétaire, l'Etat doit pouvoir rétablir la confiance rompue auprès des assujettis afin d'encaisser correctement et lui permettre de couvrir les dépenses publiques.

A ce titre, plusieurs facteurs doivent intervenir pour la mise en œuvre du recouvrement de cette vignette à Kinshasa, notamment : la confiance, la sincérité de la déclaration (comme l'a attesté Lambert Thierry, en France et en Turquie, comme dans la plupart des

40 Voir note 40.

41 Voir DERUEL, note 35, p. 27.

42 Voir BUABUA wa KAYEMBE Mathias, « Traité de droit fiscal congolais », Kinshasa, 1993, p.256.

43 Voir SAIDJ Luc, « Finances publiques », $4^{\text {ème }}$ édition, Paris, 200, p. 363. 
Etats moderne à travers le monde, l'essentiel des impôts repose sur le principe déclaratif ${ }^{44}$ ), la régularité du contrôle fiscale, l'application correcte du principe de déductibilité des charges, etc. C'est en ceci que cet impôt pourra contribuer aux édits budgétaires de la ville province de Kinshasa, et par ricochet, au budget de l'Etat congolais.

De ce fait, à tort ou à raison, l'on constate une apathie dans le chef des agents du fisc, pareille attitude ne pourrait avoir que deux remèdes : la motivation des agents par un paiement correspondant à leurs prestations et l'institution de sanctionnes exemplaires pour ceux d'entre eux dont la persistance de la cupidité et autres pratiques contra legem seraient constatées dans leur chef et prouvées par le filmage rendu possible par les caméras de surveillance ou caméras trottoir.

\section{L'instauration d'un système fiscal reformé, numérisé et plus incitatif que répressif:}

Le système fiscal Congolais dans son ensemble a été déclaré répressif. A ce sujet, Mathias BUABUA wa KAYEMBE note que : ce système ne conseille pas et frappe directement le contribuable fautif sans une confrontation préalable. Par opposition au terme répressif, on peut évoquer le terme incitatif. Pour sa part, Jean-Marie MONIER estime que : " les incitations fiscales sont utilisées pour décourager les activités nuisibles ou encourager les activités socialement et économiquement appréciées de manière positive $\mathrm{e}^{45}$.

C'est qui nous pousse à conseiller à l'autorité provinciale de Kinshasa de pouvoir alléger ou d'humaniser certaines mesures dans le recouvrement de la vignette afin de permettre aux contribuables de pouvoir s'en acquitter.

Ainsi, l'impôt peut être utilisé pour encourager, par exemple, les investissements dans certains secteurs particuliers ${ }^{46}$ tels que le transport à Kinshasa qui connaît d'énormes difficultés. Or, le système fiscal Congolais n'offre pas ce genre de dispositif incitatif. Bien au contraire, il est érigé sur le paiement sans condition et considération de certains éléments de la capacité contributive des contribuables.

C'est cette situation qui ouvre notamment la porte à des différentes tracasseries dont font l'objet en permanence les contribuables de l'impôt sur les véhicules lors du versement voire lors de l'obtention de la vignette.

\section{CONCLUSION}

Eu égard à ce qui précède, il importe de relever qu'aussi longtemps que le système físcal Congolais sera plutôt qualifié d'auto liquidatif et auto déclaratif c'est-à-dire accordant la

44 Voir THIERRY Lambert, «Le contribuable face à l'administration fiscale : psychologie et sciences administratives », p. 104, disponible in http://www.u.picardie.fr.

45 Voir MONIER Jean-Marie, "La politique fiscale : objectifs et contraintes, les cahiers français Documents d'actualité », la documentation française, 2008, p. 4.

46 Voir BUABUA wa KAYEMBE Mathias, note 38, p.88. 
possibilité pour le contribuable de calculer et de déclarer seul l'impôt à payer; le rendement de l'impôt en RDC demeurera tributaire du recensement efficient du contribuable.

De plus, il faudrait penser au renforcement de la lutte contre le flux financier illicite : l'évitement fiscal, l'évasion fiscale, l'injustice fiscale, les paradis fiscaux, etc. Sans omettre la nécessité d'assurer un encadrement et une vulgarisation des lois fiscales en cette matière envers les assujettis pour une mentalité fiscale développée et la formation des agents du fisc sur l'éthique fiscale et les avantages que présente aujourd'hui notamment le numérique.

Car, nous pensons également comme Achille UMBA que : outre son rôle régalien d'alimenter le trésor public, l'impôt sert de levier de la politique économique d'une nation pour réguler : les investissements, pour stimuler le secteur économique ou favoriser l'embauche de différentes catégories de personnes, etc. ${ }^{47} \mathrm{Ce}$ qui pourrait permettre à la RDC de tirer à divers égards profit du système fiscal incitatif.

En définitive, il conviendra de retenir que la lutte à engager pour instaurer des bonnes stratégies de recouvrement de l'impôt sur les véhicules exige une meilleure connaissance des mesures à prendre en compte en référence aux principes de la bonne gouvernance et de l'Etat de droit. A défaut de quoi, il ne serait que prétentieux de s'assurer une meilleure maîtrise de recouvrement de cet impôt.

\section{BIBLIOGRAPHIE}

\section{TEXTES JURIDIQUES}

- La Constitution de la République démocratique du Congo du 18 février 2006, modifiée par la loi n 11/002 du 02 janvier 2011 portant révision de certains articles de la constitution de la République démocratique du Congo du 18 février 2006;

- Ordonnance-loi n69-006 du 10 février 1969 sur l'impôt réel;

- Ordonnance-loi n¹3/001 du 23 février 2013 fixant la nomenclature des impôts, droits, taxes et redevances des provinces et des entités territoriales ainsi que leurs modalités de répartition;

- Edit $n^{\circ} 0002 / 08$ du 22 janvier 2008 portant procédures relatives aux impôts, taxes, redevances et autres droits dus à la ville de Kinshasa;

- Edit $n^{\circ} 0005 / 08$ du 11 octobre 2008 relatif aux impôts, taxes et droits provinciaux dus à la ville de Kinshasa. 


\section{DOCTRINE}

\section{A. OUVRAGES}

- BELTRAME Pierre, La fiscalité en France, Paris, 1993;

- BOUVIER Michel, Introduction au droit fiscal générale et à la théorie de l'impôt, $11^{\text {ème }}$ édition, Paris, 2012;

- CASTAGNE Bernard, La politique fiscale, Que sais-je? Paris, 2008;

- DERUEL, Finances publiques et le droit fiscal, $6^{\text {ème }}$ édition, Paris, 1987;

- DUVERGER Maurice, Finances publiques, Collec. Thémis, Paris, 1988;

- GUY GEST et Gilbert TIXIER, Manuel de droit fiscal, Paris, 1986;

- JEZE Gaston, Cours de finances publiques, Paris, 1923-1930;

- MONIER Jean-Marie, La politique fiscale : objectifs et contraintes, Les cahiers français Documents d'actualité, La documentation française, 2008;

- SAIDI Luc, Finances publiques, $4^{\mathrm{ème}}$ édition, Paris, 2003;

\section{B. ARTICLES DE REVUE}

- BUABUA WA KAYEMBE Mathias, "Le droit fiscal congolais et son avenir », in Revue de la Faculté de droit (UPC), $2^{\text {ème }}$ numéro, 2001;

- EROY Marc, "La sociologie fiscale, socio-logos », in Revue de l'association française de sociologie, disponible in http://socio-legos.revue.org;

- UMBA Di MAMONA Achille, «Piste pour une fiscalité en République démocratique du Congo ", in Revue africaine de la démocratie et de la gouvernance (RADG), African journal of democracy and gouvernance (AJGD), Institut pour la démocratie, la paix et le développement en Afrique (IDGPA), Volume I, n²4, 2014;

\section{NOTES DE COURS}

- KOLA GONZE Roger, « Droit fiscal congolais », Université de Kinshasa, 2019-2020;

- NTUMBA MUSUKA Zacharie-Richard, Finances publiques, faculté de droit, Université de Kinshasa, 2017-2018.

\section{AUTRES DOCUMENTS}

- «Guide fiscal de l'artiste plasticien », la documentation française, Paris 1987;

- Dictionnaire le Larousse, édition 2016;

- L'extrait du témoignage du journalise Danny MANGBAU, disponible in https://Scooprd c.net;

- Extrait du discours du Jean-Paul BILANGA N'OSHUM, directeur général de la Direction Générale des Recettes de Kinshasa, disponible in https://www.google.com/lepotenti elonline 
- THIERRY Lambert, « le contribuable face à l'administration fiscale : psychologie et sciences administratives », in http://www.u.picardie.fr. 